\title{
Toys in physics lectures and demonstrations-a brief review
}

\author{
J Güémez ${ }^{1}$, C Fiolhais ${ }^{2}$ and M Fiolhais ${ }^{2}$ \\ ${ }^{1}$ Departamento de Física Aplicada, Universidad de Cantabria, E-39005, Santander, Spain \\ ${ }^{2}$ Departamento de Física and Centro de Física Computacional, Universidade de Coimbra, \\ P-3004-516 Coimbra, Portugal \\ E-mail: guemezj@unican.es, tcarlos@teor.fis.uc.pt and tmanuel@teor.fis.uc.pt
}

\begin{abstract}
The use of toys in physics teaching is common. This brief review of the physics of toys intends to show that they are not only very useful in lectures and demonstrations in order to motivate students but also very interesting from a scientific point of view. However, since their physics is sometimes too cumbersome, the effect can be the opposite. We call attention to some subtleties of toys used in physics or in general science teaching.
\end{abstract}

An analysis of the pedagogical literature shows that toys have been widely used to illustrate and to discuss various physics topics [1] in physics lectures and demonstrations for both students and popular audiences [2]. In fact, toys like old balancers [3] or modern plasma balls [4] (figure 1) provide an amusing way to teach physics to a range of audiences. However, the explanation of their working may be quite involved, so the teacher should master the underlying subjects in order not only to identify and convey the most important messages, but also to provide correct answers to the audience's questions. In some cases the use of toys is not recommended, to avoid difficulties and confusion, which could discourage the audience.

In this article we discuss a selection of toys with interesting physics, making remarks on scientific aspects and giving references, mainly recent, for deeper study by both educators and students.

Spinning tops are among the most ancient toys. There is a huge variety of them. The simplest spinning top spins nicely until it stops and falls down - the stability of its vertical (or almost vertical) spin axis is basically explained by the laws of angular momentum and energy conservation [5] (figure 2, left). The spinning equilibrium of a gyroscope on a narrow support is more spectacular, although its physics is essentially the same. It is quite easy to start these objects spinning and explain the motion in general terms, but the detailed equations of a top or of a gyroscope are very challenging and may only be grasped at a university level [6].

The magnetically levitating top, also called a Levitron, has been widely discussed in the literature (figure 2, right) [7]. It consists of a small top, and a large base with strong magnets hidden in it. By spinning the top and placing it carefully a few centimetres above the base (which produces the field), it will remain floating for a long time. Air friction gradually reduces its angular velocity so that, after some time, the top falls down.

An interesting spinning top contains some balls inside a transparent hemisphere (figure 3). When the top starts to rotate, the balls follow the rotation going around, at the edge of the hemisphere.

Rotating devices sometimes exhibit a counterintuitive behaviour. One of the most bizarre is that 

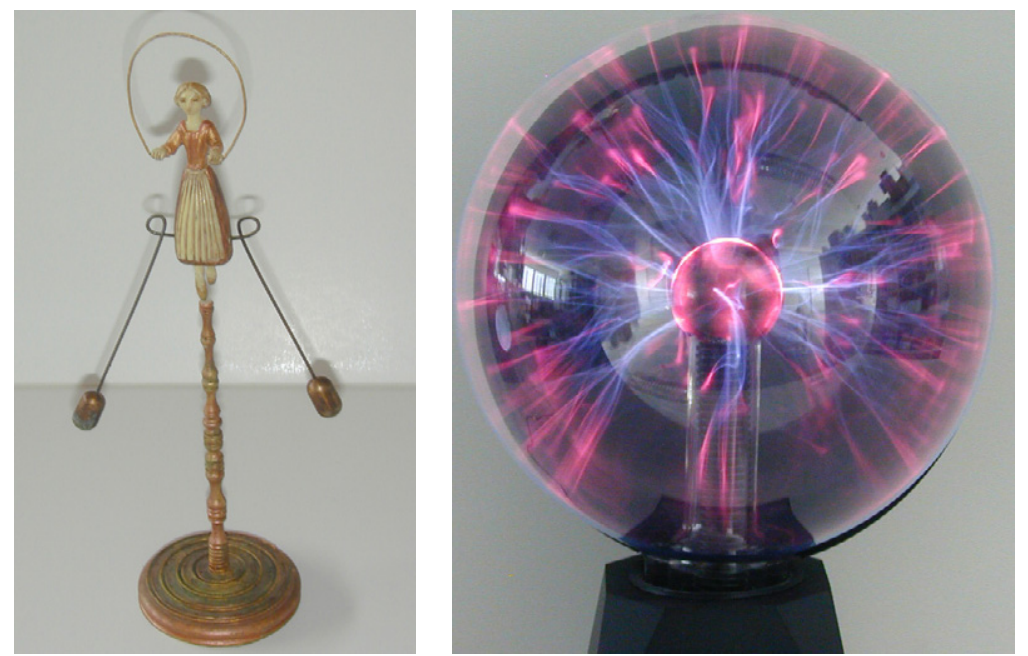

Figure 1. The old balancing toy (left) does not fall down, since a torque always acts towards equilibrium. In the modern plasma ball (right), electrons emitted by a metallic surface at the centre collide randomly with excited atoms of a gas at low pressure.
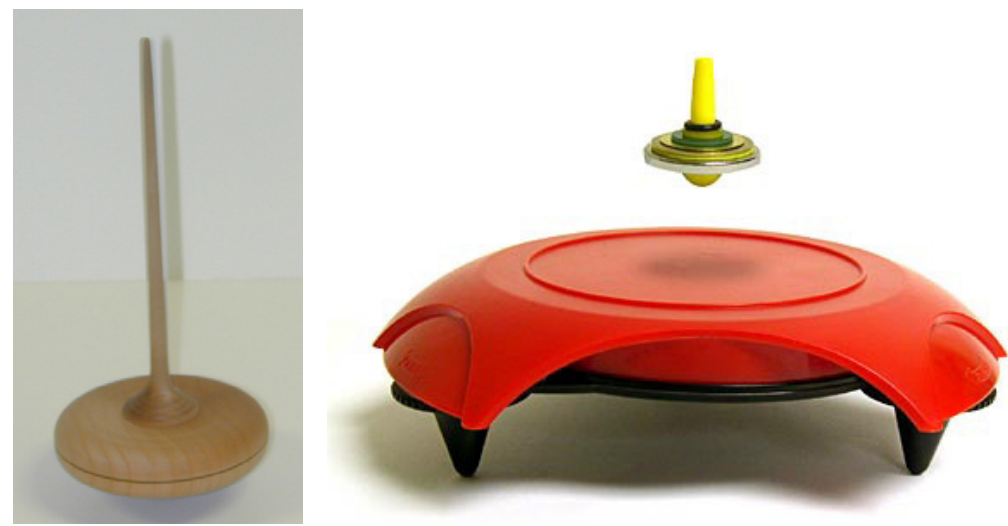

Figure 2. A wooden spinning top (left) and the Levitron (right, courtesy ThinkGeek, www.thinkgeek.com), which is a modern top.

exhibited by the so-called tip-top (or tippe top), which appears in a variety of models [8]. This top, similar to a mushroom (figure 4), flips over on its stem after spinning for a while. Although a detailed explanation of the flip-over of tip-tops is not simple, we note that rotation is only stable around the symmetry axis. Similar behaviour is exhibited by a hard-boiled egg (or wooden egg) when rotating around its stable static equilibrium position. When a hard-boiled egg is rotated quickly enough starting from its static equilibrium position (the egg lying horizontally), it will elevate its centre of mass while continuing to rotate around its symmetry axis. A raw egg does not show this behaviour due to the liquid inside, so this feature may be used to distinguish the two eggs (more easily, we may press a finger on a rotating egg: a hard-boiled egg stops immediately, while a raw egg continues rotating due to the liquid). It is the friction with the bottom surface and the position of the centre of mass below the centre of curvature which causes the top or the egg to rise.

Also bizarre is the rattleback, which also appears in different sizes and shapes (figure 5). This has the general shape of a small boat and, 


\section{Toys in physics lectures and demonstrations—a brief review}
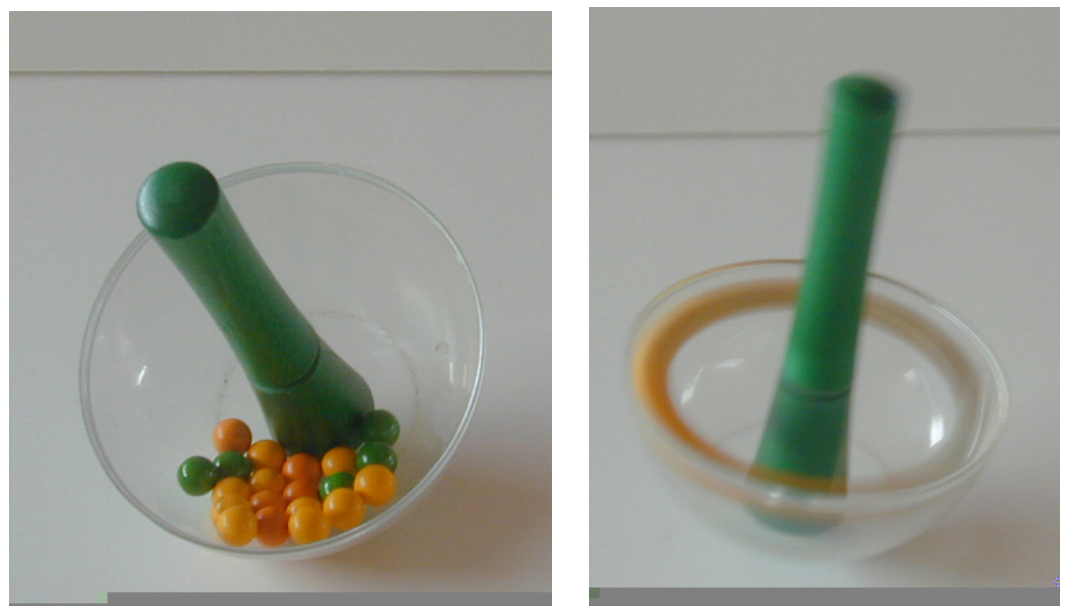

Figure 3. Spinning top with some small balls inside a covered hemisphere (left). When the top rotates, the balls go up to the edge (right).
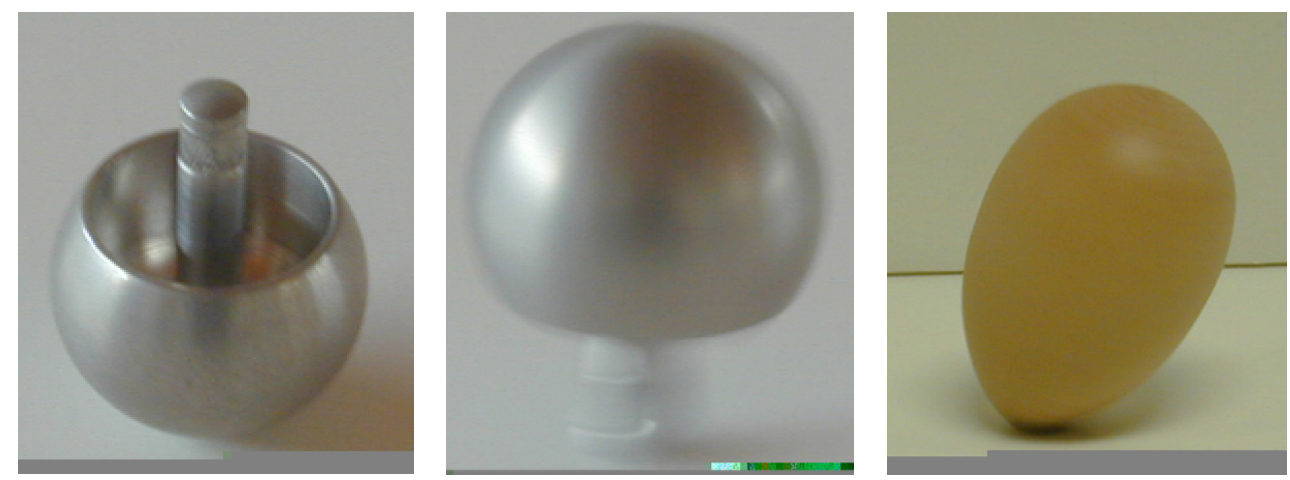

Figure 4. A tippe top is a section of a sphere with a rod (left). After spinning on its rounded body it flips over and continues rotating around its elongated stem (middle). The same happens with a rotating wooden egg (right).

after spinning for some time in one direction, it reverses its motion until it eventually comes to rest [9]. This phenomenon is due to the asymmetric form of the object, the full physical explanation being quite involved. We may, however, say that the system rattles back due to unbalanced moments.

Different pendulums-including balancers (like that of figure 1), which are nothing but physical pendulums in stable equilibrium [10] form another curious set of toys. A suspended oscillating mass tied to a string, or a ruler or a rigid body pivoted to oscillate around an axis passing through a hole in the object, are other examples of pendulums. A toy based on the energy interchange of a pendulum is the well-known Newton's cradle (figure 6). The balls collide, following the laws of

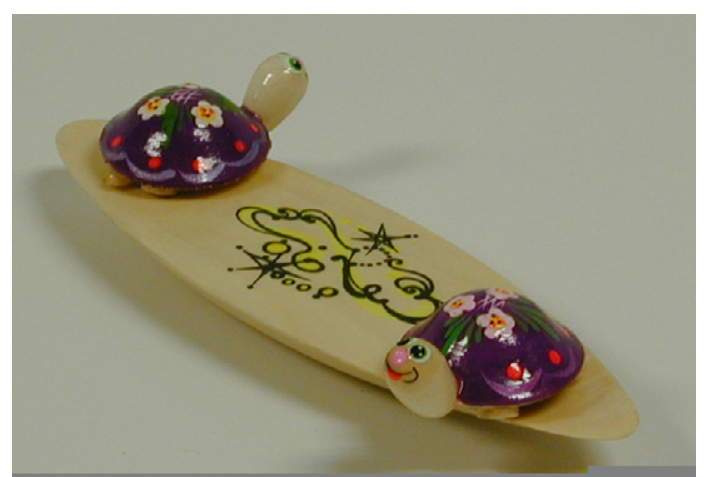

Figure 5. A wooden model of a rattleback. When rotating clockwise it rotates like any other rotating object. When rotating counterclockwise, i.e. in the opposite direction to the turtles, it eventually stops, rattles and begins to rotate backwards. 


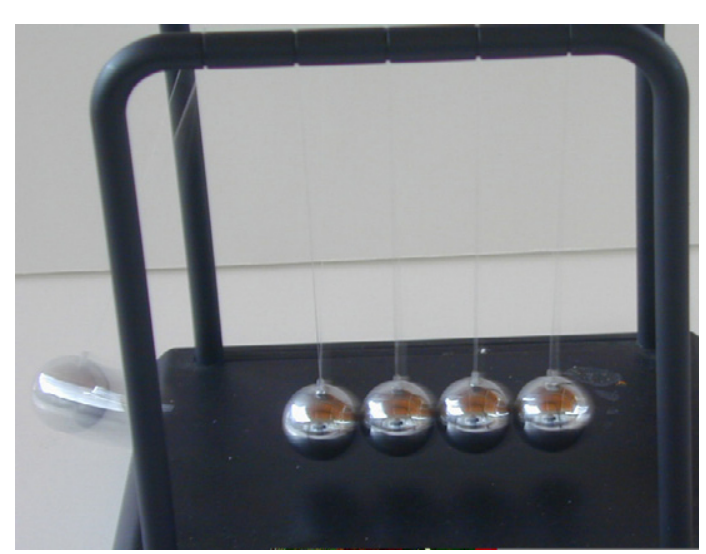

Figure 6. Newton's cradle, which follows the original Gravesande design for studying collisions. One or more balls at one side collide with the intermediate balls, causing the same number of balls to rise at the other side.

conservation of linear momentum and mechanical energy, and eventually stop. It is well known that the number of balls which rise is equal to the number of colliding balls, but it is less known that the force between the spheres does not obey Hooke's law (it is proportional to the deformation to the power 3/2).

Also challenging is the behaviour of socalled non-smooth dynamical systems like the notched stick, the tumbling toy, the descendent woodpecker, or the walking duck (figure 7) [11], all of them with a complex dynamics but understood in a simple form through conservation principles and application of the formalism of moments.
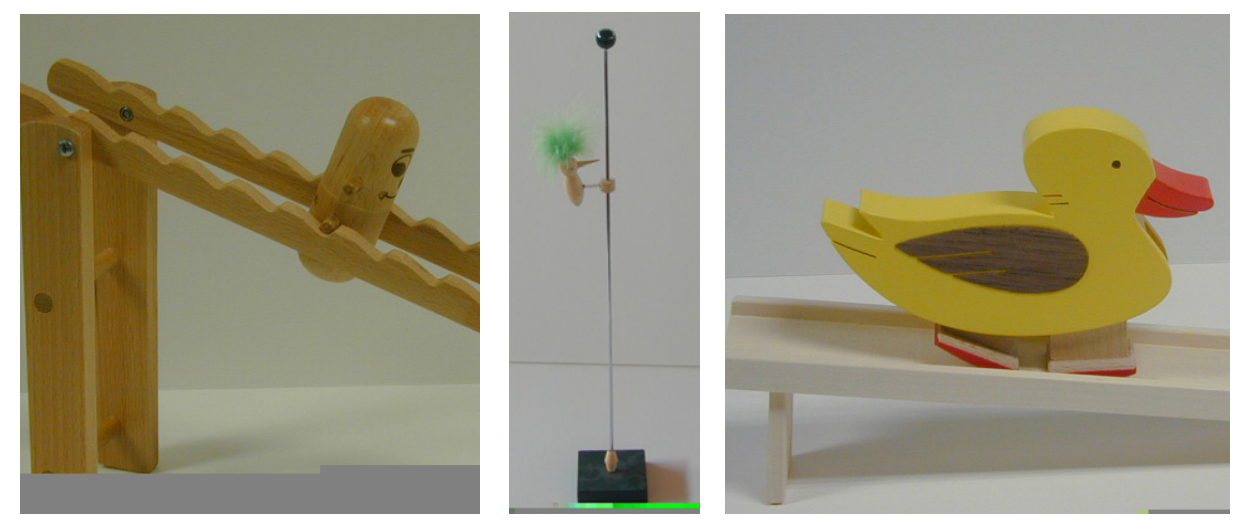

Figure 7. Non-smooth dynamical toys: tumbling toy (left), descendent woodpecker (middle), and waddling duck (right). 


\section{Toys in physics lectures and demonstrations-a brief review}
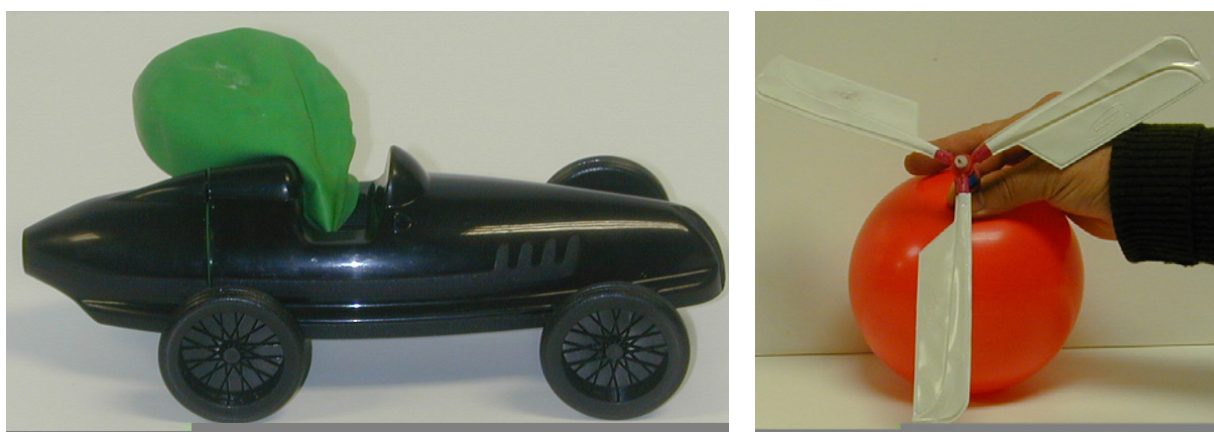

Figure 9. Toys with balloons for demonstrating the conservation of linear momentum and Newton's third law: car (left) and helicopter (right).
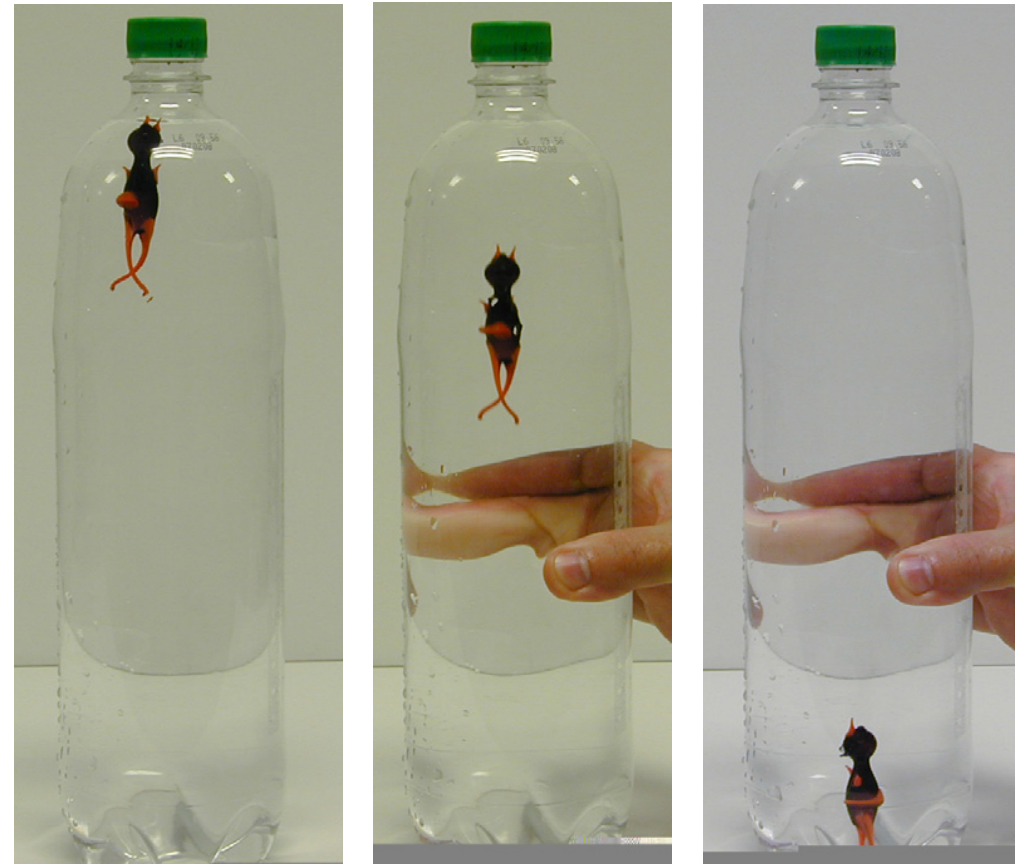

Figure 10. Cartesian diver. A hollow glass sphere, decorated as a little devil, and with a hole at the end of its tail, retains an air bubble (left). When the flexible bottle is gently squeezed (middle), the bubble size decreases, and the buoyancy is not sufficient to balance the weight of the devil, which sinks (right).

in many cases, involved physics if we look carefully at them. The so-called Cartesian diver [17] provides a nice example (figure 10). The diver has an air bubble inside: when the pressure is increased (usually by squeezing the plastic bottle which contains the diver), the bubble size decreases and the reduced upthrust is responsible for the diver sinking. It is not widely known that this toy exhibits a fold catastrophe behaviour which may be used to demonstrate the application of mathematical catastrophe theory in physics [17].

Galileo's thermometer (figure 11) is a kind of Cartesian diver in which the divers are closed spheres containing a coloured fluid and the temperature replaces the pressure as the control parameter [18]. The external temperature is indicated by the lowest floating sphere. At a higher temperature, this sphere sinks and the next sphere above occupies the lowest floating position, 

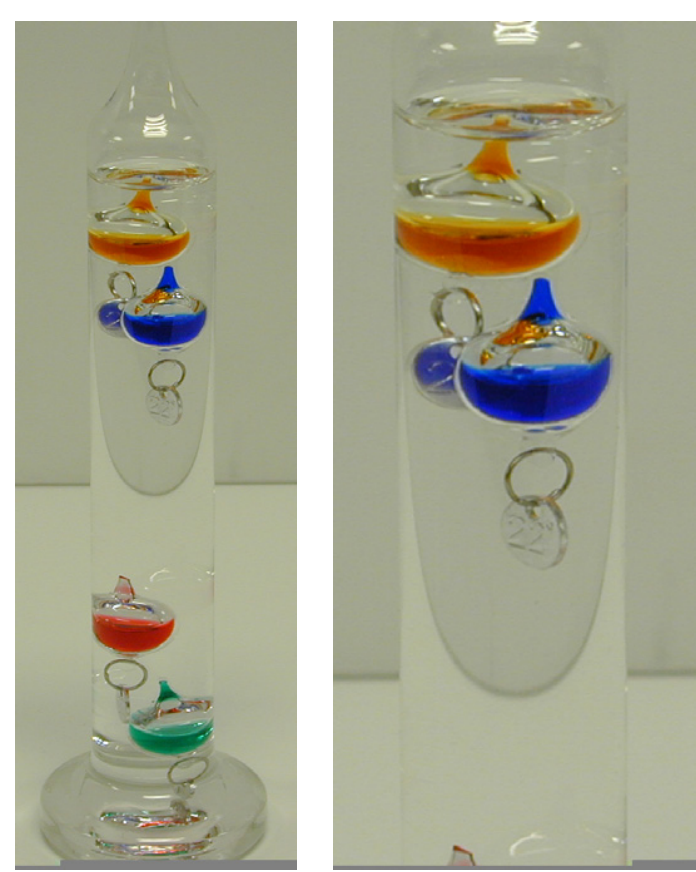

Figure 11. Galileo's thermometer. The balls inside a liquid, containing a coloured fluid, sink or float according to the value of the room temperature: they are a kind of Cartesian diver. The temperature is indicated by the lowest ball which still floats.

indicating the new room temperature. Galileo's thermometer is based on the change of the liquid density with temperature. Of course the accuracy of the measurement is very limited.

There are many other intriguing toys based on fluids [18]. The lava lamp (figure 12), which is a visually appealing device, is another example. The wax at the bottom of the lamp initially has a larger density than the alcohol above. When the wax heats up, it expands, decreasing its density. It rises, reaching the top, cools down, and then descends. This kind of convection current may be difficult to explain in detail, yet is simple in principle.

Another example of toys based on fluids consists of a ball suspended on an air stream (figure 13): some models use a tube and a table tennis or polystyrene ball. The explanation of the stability in the case of a vertical flux requires the use of Bernoulli's law as well as Newton's third law. A ball can also be suspended in an inclined air stream, but this is more difficult to explainthe Coanda effect provides an explanation that is easier to understand [19].

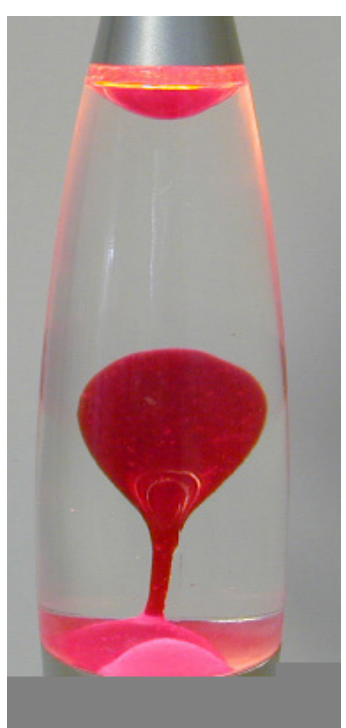

Figure 12. Lava lamp. Thermal expansion, Archimedes buoyancy, and surface tension are responsible for the amazing dynamical processes observed.

Toys which transform heat into kinetic energy are the counterpart of the mechanical ones mentioned above, such as spinning tops or pendulums, which lose mechanical energy. The thermal swing (figure 14) uses the difference between the temperature of a candle and room temperature to produce a periodic motion. The candle heats a spring attached to a semicircular metallic rod and to a body. When the bimetallic spring [20] is heated its curvature decreases and it moves the balance across. Then, the spring also moves, coming out of the flame, and cools down. The balance ball moves again, now in the opposite direction, and the spring returns to the flame. This is a simple thermal engine: both flame heating and spring cooling in the air are necessary to create the cycle. A fraction of the energy given by the candle to the spring is dissipated in the air.

The well-known Crookes radiometer [21] is another example of a thermal engine (figure 15). It works with very low air pressure. Students often believe that the blades are turned due to the 'pressure of light'. This is not the right explanation, because even the black faces move in the direction of the incident light. The usual explanation of its working is that the air near the black surface is at a temperature slightly higher than the air close to the white surface. This higher 

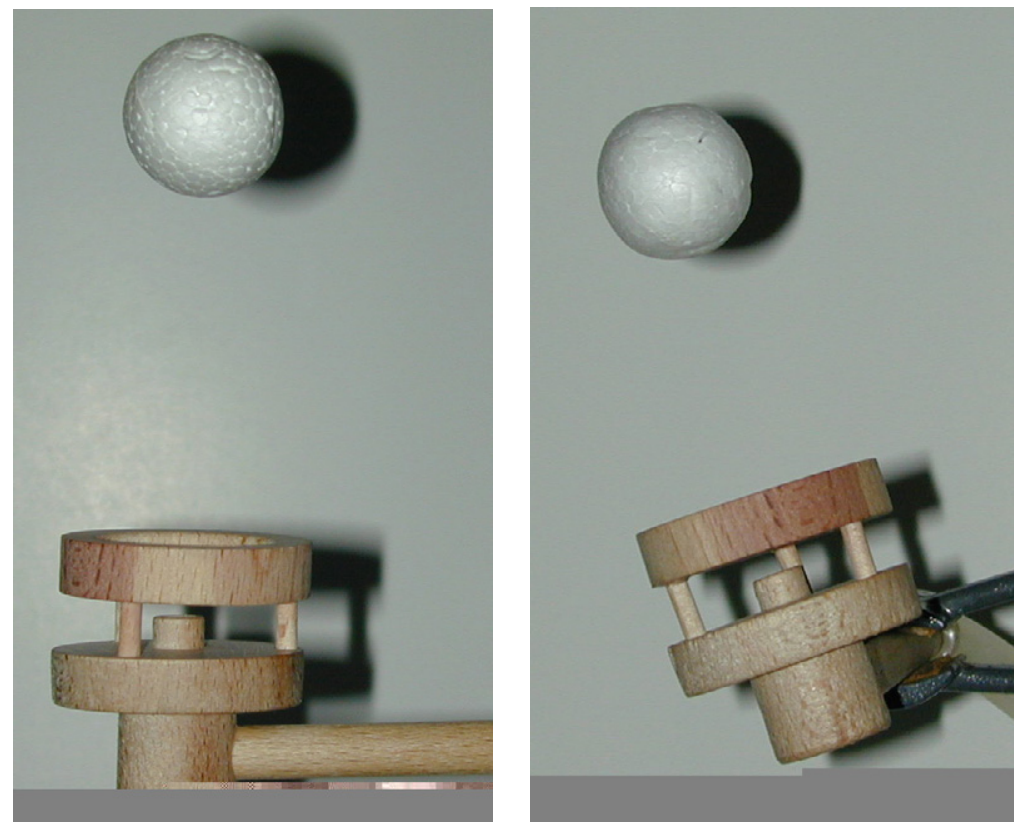

Figure 13. Levitating ball in a vertical air flow (left), in accordance with Bernoulli's law, and in an inclined one (right).
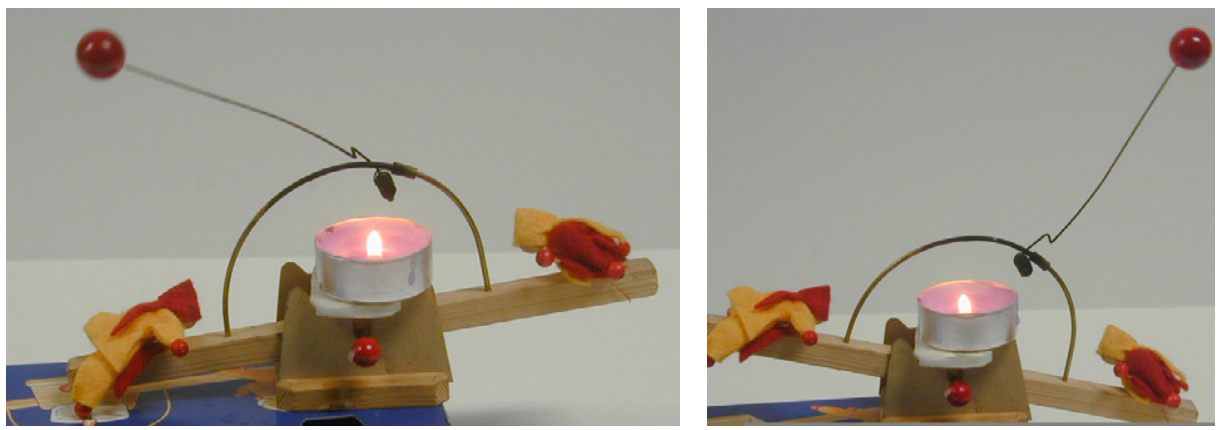

Figure 14. A thermal swing. Heating the bimetallic spring with the candle causes the wooden sphere at the end of the rod to flip position (left). The system moves after the spring cools down (right); the wooden sphere returns to its initial position and the cycle repeats.

temperature means that the air molecules collide with the rim of the black surface more violently, which pulls this surface away from the light. When both sides of the blades are painted, alternately black and white, the sail rotates more slowly than in the case when one side is black and the other is a polished metal, since the temperature difference is smaller in the first case. The radiometer can also be made to rotate backwards when placed in a cool environment (e.g. a refrigerator).
The drinking bird (figure 16) is another example of a thermal engine: it transforms a chemical potential difference into mechanical work [22] without a chemical reaction. If the air is not saturated, some water evaporates in the bird's head. During this evaporation the head temperature decreases and the same happens with the vapour pressure of the gas inside the bird's body. This gas, which is at a higher pressure, elevates the liquid. As the internal liquid rises, the centre of mass moves and the bird eventually falls 

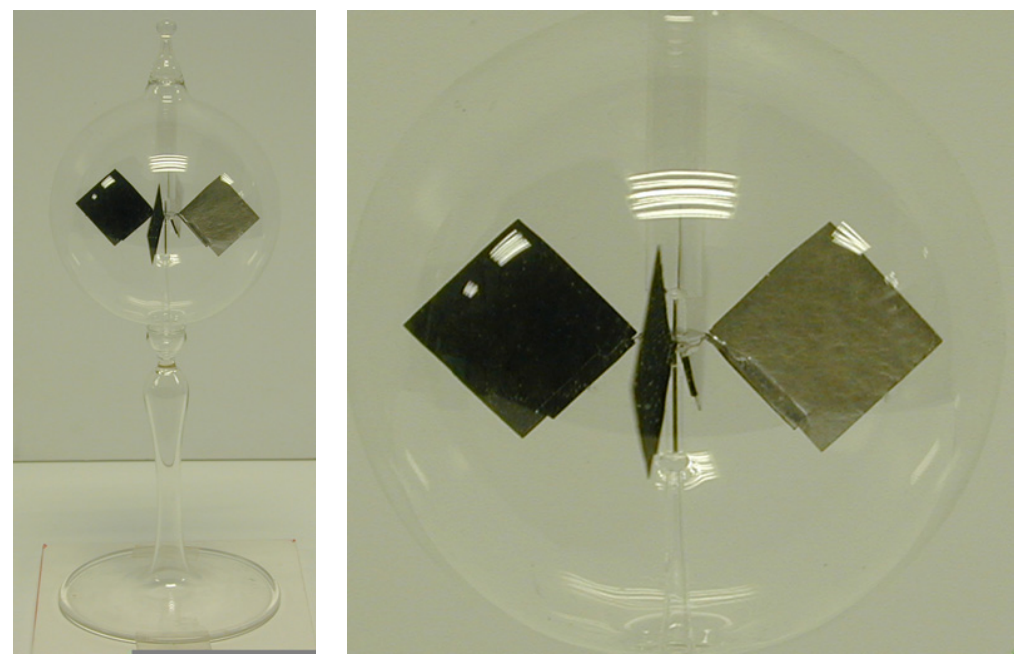

Figure 15. Crookes radiometer. The rotation is induced by light, but is due to the larger impact of air molecules close to the black paddles.

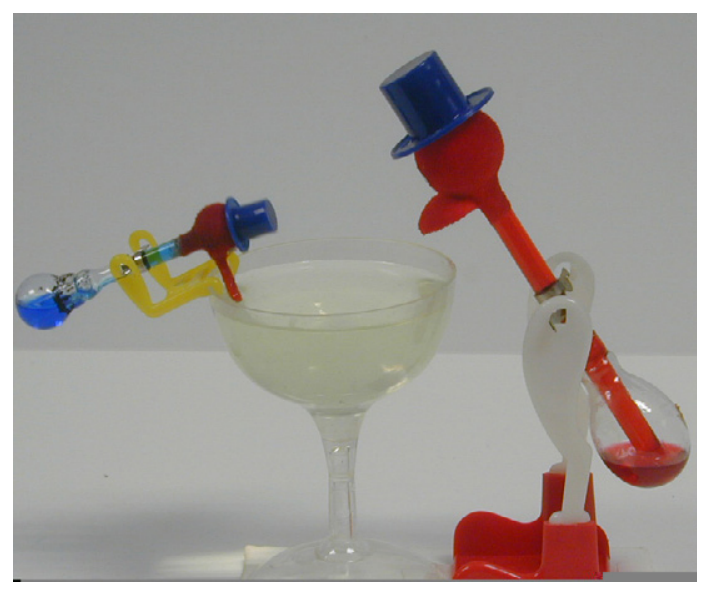

Figure 16. Two drinking birds. The water in the bird's head evaporates, the internal liquid rises, the bird inclines and 'drinks', the liquid returns afterwards to the body, and another cycle starts. The smaller bird 'drinks' more quickly.

down. At this stage the liquid comes down, and the process repeats itself. Of course, the liquid inside the bird should have particular thermodynamical properties such as a normal boiling point close to room temperature (for instance, the boiling point for the commonly used $\mathrm{CH}_{2} \mathrm{Cl}_{2}$ is $40^{\circ} \mathrm{C}$ ).

Another old and intriguing toy based on heat is the chop-chop boat [23] (figure 17). The impelling oscillatory liquid motion outside and inside its narrow tubes transforms the heat coming from a candle flame into kinetic energy associated to the boat's motion. The tubes are filled up with water and immersed. When the candle is ignited, the water vaporizes and the vapour forces the water to come out of the tubes so that the boat moves forward. When it cools, the vapour pressure decreases and the atmospheric pressure forces the water to re-enter the tube, repeating the cycle. The vibration of a thin metallic plaque during the motion is responsible for the chop-chop noise, but this is an add-on unrelated to the main mechanism.

Electric and magnetic toys have a peculiar charm. Even a simple electric motor shows a curious working: the coil rotates due to its immersion in a magnetic field, provided by a fixed magnet. The Lorenz force makes the coil rotate. A simpler electromagnetic motor [24] can be constructed, in which the magnet acts like a fan. To build this so-called homopolar motor, we only need a strong magnet (for example, neodymium), a screw, a battery, and a copper cable (figure 18). The Lorenz force within the magnet makes the magnet rotate at high speed.

Magnets are very appealing to everybody. The magnetic chaotic pendulum (figure 19) consists of the motion of a metal sphere in the field originated by a peculiar location of magnets, which are normally hidden. It may be used to introduce in a visual way the theory of chaos: very close initial conditions lead to completely different 
Toys in physics lectures and demonstrations-a brief review

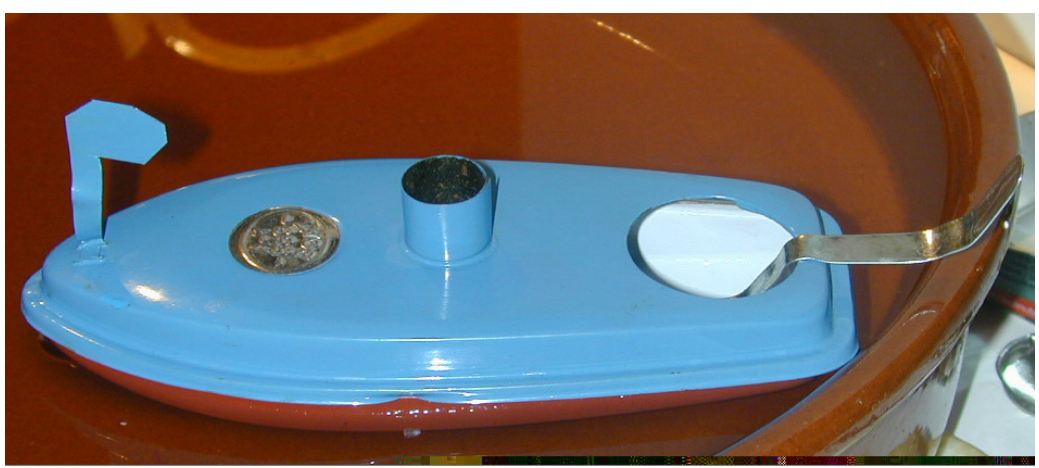

Figure 17. The chop-chop boat moves forward due a built-in heat engine.
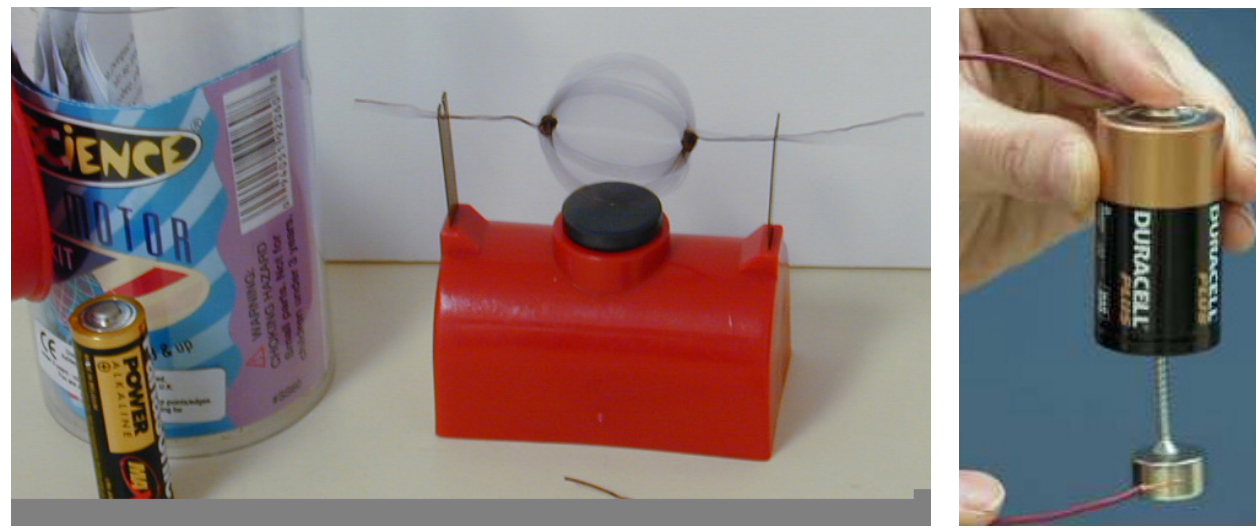

Figure 18. Electromagnetic motors. A simple electric motor: when a current provided by a battery (not shown) flows across the coil, located above a magnet, the coil rotates (left). In the homopolar motor, the magnet is rotating beneath (right).

trajectories, although the behaviour is strictly ruled by Newtonian laws [25]. The Gauss gun is another interesting toy, which demonstrates how magnetic energy can be converted into kinetic energy.

Many magnetic toys show magnetic levitation like the above-mentioned Levitron. Magnetic fields produced by graphite plates, which are diamagnetic, may help to create a stable equilibrium (stable potential minimum) for a magnet, which floats under a ferrite magnet [26] (figure 20). Although this is not exactly a toy, it allows a curious experiment which is always an attraction to children. We note that the physics of diamagnetism as well as that of ferromagnetism is essentially quantum mechanical.

Coming back to the plasma lamp (figures 1 and 21), this can only be understood on the basis of modern physics. At the centre of the ball a very high voltage is produced by a Tesla coil. The

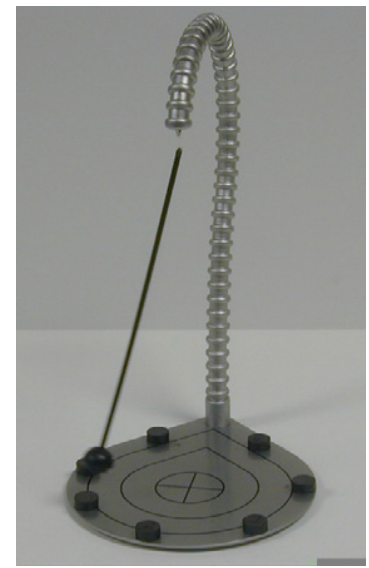

Figure 19. Chaotic magnetic pendulum: the ball, subject to a non-harmonic potential, performs a deterministic chaotic motion, i.e., a motion very sensitive to the initial conditions, although ruled by simple laws. 


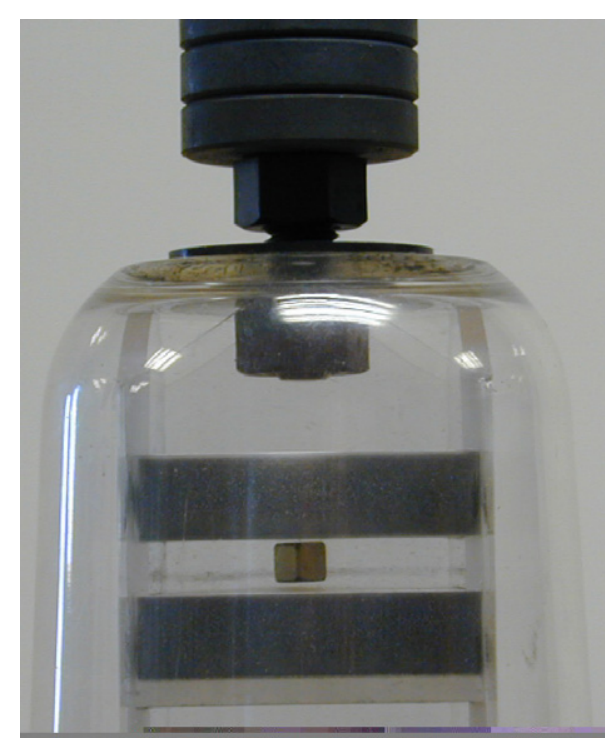

Figure 20. Levitating magnet (cube) in between two diamagnetic graphite plates and with ferrite magnets at the top. The unstable equilibrium between the magnetic force (up) and gravitation (down) is stabilized by the diamagnetic material

observed light is emitted when the atoms capture electrons, returning to their ground state. Of course, young people playing with this lamp do not need to know that quantum mechanics has a role in its working, but higher level physics students certainly should know that.

Toys related to optics, like kaleidoscopes which use mirrors [27], are based only on the

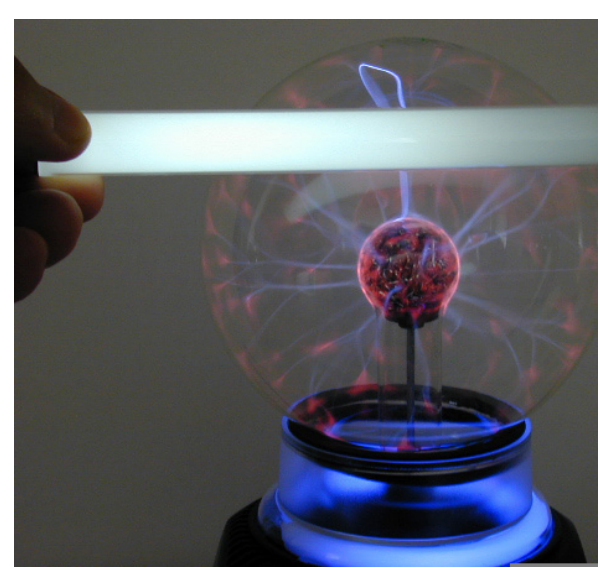

Figure 21. A plasma lamp is a toy combining classical and modern physics. The fluorescent tube above is illuminated due to the plasma lamp.

Snell-Descartes law of reflexion, and have always been popular. Using sections of spherical mirrors it is possible to obtain a surprising real mirage (figure 22): when an object, such as a small plastic pig, is hidden at the centre of the bottom mirror, one sees two images near the apex of the top mirror (note that the images are real).

In this review on the physics of toys we have sketched the behaviour of a selected set of gadgets (there are, of course, many more!), emphasizing not only their educational use but also some of the associated difficulties.

With these devices we may see that physics has multiple faces and that the physical
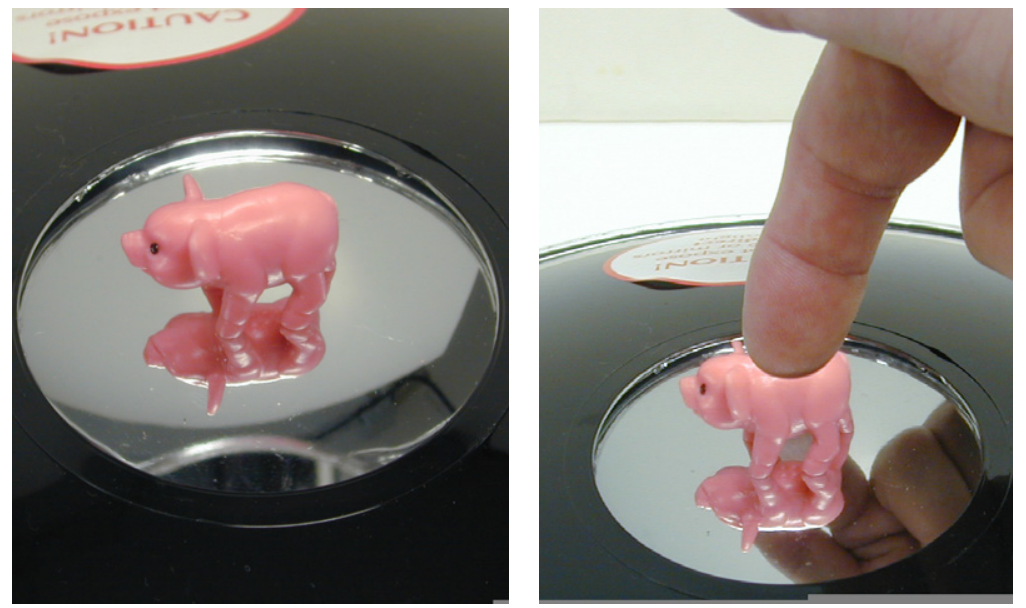

Figure 22. Mirage: the floating image of a little pink pig, by the mirage, cannot be touched. It is interesting to ask what happens if a torch or laser pen is shone at the image. 
Toys in physics lectures and demonstrations-a brief review

explanation of simple toys may require, at the same time, concepts of mechanics, thermodynamics, electromagnetism, quantum physics, etc. The same occurs in general in the natural world: the description of a single phenomenon may involve various areas of physics.

We believe that toys may play a role in introducing young people to science and to the scientific method. In particular, after an experiment with a toy has been performed, questions may be raised such as 'and what happens if ...'? For instance, what happens if the Cartesian diver is $1 \mathrm{~m}$ deep instead of only $20 \mathrm{~cm} \mathrm{[17]?}$ And what happens if the body of the drinking bird is painted black and illuminated (the socalled 'sunbird') [22]? The physics of toys can also be used to encourage advanced students to develop small research projects. According to our own experience, the construction of toys in the laboratory is a valuable occasion to learn hands on. Students may realize that reality is richer than the theory appearing in textbooks.

Finally, we stress that toys, interesting as they may be, are not a pedagogical panacea. On the contrary, they are not recommended if their basic principles are too difficult for the intended audiences. Though the balancer, the Newton's cradle, the balloon car, the electric motor, and the optical mirage are based on simple physics, this is not the case with spinning tops (in particular the rattleback), the tumbling toy, Crookes radiometer and the plasma lamp. It is the instructor's task to select what to show and what to say on each occasion.

Received 13 February 2008, in final form 3 September 2008 doi:10.1088/0031-9120/44/1/008

\section{References}

[1] Aref H, Hutzler S and Weaire D 2007 Toying with physics Europhys. News $\mathbf{3 8} 23$

Featonby D 2005 Toys and physics Phys. Educ. 40537

[2] Shugart C G 1976 Saturday science Phys. Teach. 1491

Willis J and Kirwan D F 1976 Physics demonstrations for the public Phys. Teach. 14210

[3] Fort J, Llebot J E, Saurina J and Suñol J J 1998 A counterintuitive toy: the bird that never falls down Phys. Educ. 3398

Turner R C 1987 Toys in physics teaching: balancing man Am. J. Phys. 5584
[4] Guilbert N R 1999 Deconstructing a plasma globe Phys. Teach. 3711

[5] Bolina O 2000 The precessing top Phys. Teach. 38312

[6] Case W 1977 The gyroscope: an elementary discussion of a child's toy Am. J. Phys. 451107

[7] Taylor M 2001 Curiosity: the Levitron Phys. Educ. 36 259-60

[8] Soodak H 2002 A geometric theory of rapidly spinning tops, tippe tops, and footballs Am. J. Phys. 70815

Cohen R J 1977 The tippe top revisited Am. J. Phys. 4512

Sasakia K 2004 Spinning eggs—which end will rise? Am. J. Phys. 72782

[9] Edge R D and Childers R 1999 Curious celts and riotous rattlebacks Phys. Teach. 3780

Crane H R 1991 The rattleback revisited Phys. Teach. 29278

[10] Bowen J M 1982 Slinky oscillations and the notion of effective mass Am. J. Phys. 501145

Cromer A 1995 Many oscillations of a rigid rod Am. J. Phys. 63112

Gavenda J D and Edgington J R 1997 Newton's cradle and scientific explanation Phys. Teach. 35411

Ehrlich R 1996 Experiments with 'Newton's cradle' Phys. Teach. 34181

[11] Jax Mulder D P 1980 Children's toys Phys. Teach. 18 134-5

[12] Aguirregabiria J M, Hernández A and Rivas M 2007 Falling elastic bars and springs Am. J. Phys. 75583

Toepker T P 2004 Center of mass of a suspended slinky: an experiment Phys. Teach. $\mathbf{4 2} 16$

[13] Van Hook S J 2005 Teaching potential energy functions and stability with slap bracelets Phys. Teach. $\mathbf{4 3} 425$

Ng Y-K, Mak S-Y and Chung C-M 2002 Demonstration of Newton's third law using a balloon helicopter Phys. Teach. $\mathbf{4 0} 181$

[14] Finney G A 2000 Analysis of a water-propelled rocket: a problem in honors physics Am. $J$. Phys. 68223

[15] de Sousa C A and Pina E P 1997 Aspects of mechanics and thermodynamics in introductory physics: an illustration in the context of friction and rolling Eur. J. Phys. 18334

[16] Kires M 2007 Archimedes' principle in action Phys. Educ. 42484

Lan B L 2001 An ice-cube puzzle Phys. Educ. 3675

[17] Güémez J, Fiolhais C and Fiolhais M 2003 A demonstration apparatus for the Cartesian diver Phys. Teach. 41495

Güémez J, Fiolhais C and Fiolhais M 2002 The Cartesian diver and the fold catastrophe Am. $J$. Phys. 70710

[18] Nickas G D 1989 A thermometer based on Archimedes' principle Am. J. Phys. 57845 
[19] McDonald K T 2000 Levitating beachballs Am. J. Phys. 68388

Weltner K 1987 A comparison of the explanations of the aerodynamic lifting force Am. J. Phys. 5550

[20] Hewson P W 1975 The bimetallic strip-a quantitative experiment Phys. Teach. 13350

[21] Crawford F S 1985 Running Crookes radiometer backwards Am. J. Phys. $\mathbf{5 3} 1105$

Coisson R and Rancan E 1979 Quantitative use of a Crookes radiometer Phys. Educ. 1458

[22] Güémez J, Valiente R, Fiolhais C and Fiolhais M 2004 A big sunbird Phys. Teach. 42307

Güémez J, Valiente R, Fiolhais C and Fiolhais M 2003 Experiments with the drinking bird Am. J. Phys. 711257

[23] Finnie I 1963 Physics in a toy boat Am. J. Phys. 31289

Miller J S 1958 Physics in a toy boat Am. J. Phys. 26199

[24] Featonby D 2007 An even simpler version of the neodymium motor Phys. Educ. 42236

Featonby D 2006 Inspiring experiments exploit strong attraction of magnets Phys. Educ. 41292

[25] Oliver D 1999 A chaotic pendulum Phys. Teach. 37174

Berry D A 1982 A 'perpetual motion' toy? Phys. Teach. 20319

[26] Edge R 2003 A simple diamagnetic levitation experiment Phys. Teach. $\mathbf{4 1} 122$

Sawicki C A 2001 Small inexpensive diamagnetic levitation apparatus Phys. Teach. 39556
[27] Sieradzan A 1990 Teaching geometrical optics with the 'optic mirage' Phys. Teach. 28534

Julio Güémez has a $\mathrm{PhD}$ from the

University of Salamanca, Spain. He

joined the University of Cantabria in

1990 as professor of thermodynamics.

His primary physics interest is in

statistical mechanics and chaos theory.

He is responsible for the 'El Torreón de la

Física', a permanent exhibition of

scientific toys and interactive

experiments.

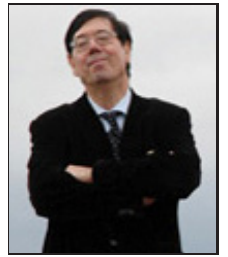

Carlos Fiolhais is a condensed matter computational physicist. He is active in science outreach and is the author of school textbooks, popular science books and science books for children. Currently, he is the Director of the General Library of the University of Coimbra, Portugal.

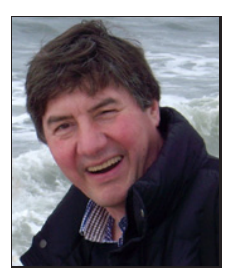

Manuel Fiolhais is a nuclear and particle physicist. He is author of several textbooks for middle and high school students. Currently, he is the Director of the Centre for Computational Physics of the University of Coimbra, Portugal, and President of the Institute for

Interdisciplinary Research of the same university. 